\title{
BMJ Open Collaborative care model for depression in rural Nepal: a mixed-methods implementation research study
}

Pragya Rimal (D) ,1,2 Nandini Choudhury, ${ }^{3,4}$ Pawan Agrawal, ${ }^{1}$ Madhur Basnet, ${ }^{1,5}$ Bhavendra Bohara, ${ }^{1}$ David Citrin, ${ }^{4,6}$ Santosh Kumar Dhungana, ${ }^{7}$ Bikash Gauchan, ${ }^{1}$ Priyanka Gupta, ${ }^{1}$ Tula Krishna Gupta, ${ }^{1}$ Scott Halliday, ${ }^{4,8}$ Bharat Kadayat, ${ }^{1}$ Ramesh Mahar, ${ }^{1}$ Duncan Maru (D) ,, ${ }^{3,4}$ Viet Nguyen, ${ }^{9,10}$ Sanjaya Poudel, ${ }^{1}$ Anant Raut, ${ }^{3,4}$ Janaki Rawal, ${ }^{1}$ Sabitri Sapkota, ${ }^{2,3}$ Dan Schwarz, ${ }^{4,11}$ Ryan Schwarz, ${ }^{4,11}$ Srijana Shrestha, ${ }^{4,12}$ Sikhar Swar, ${ }^{1}$ Aradhana Thapa, ${ }^{2}$ Poshan Thapa, ${ }^{13}$ Rebecca White, ${ }^{1}$ Bibhav Acharya ${ }^{4,14}$

To cite: Rimal P, Choudhury N, Agrawal P, et al. Collaborative care model for depression in rural Nepal: a mixedmethods implementation research study. BMJ Open 2021;11:e048481. doi:10.1136/ bmjopen-2020-048481

- Prepublication history and additional supplemental material for this paper are available online. To view these files, please visit the journal online. (http://dx.doi.org/10.1136/ bmjopen-2020-048481).

PR and NC contributed equally.

PR and NC are joint first authors

Received 30 December 2020 Accepted 03 August 2021

Check for updates

(c) Author(s) (or their employer(s)) 2022. Re-use permitted under CC BY-NC. No commercial re-use. See rights and permissions. Published by BMJ.

For numbered affiliations see end of article.

Correspondence to

Pragya Rimal;

pragya@possiblehealth.org

\section{ABSTRACT}

Introduction Despite carrying a disproportionately high burden of depression, patients in low-income countries lack access to effective care. The collaborative care model (CoCM) has robust evidence for clinical effectiveness in improving mental health outcomes. However, evidence from real-world implementation of $\mathrm{CoCM}$ is necessary to inform its expansion in low-resource settings.

Methods We conducted a 2-year mixed-methods study to assess the implementation and clinical impact of CoCM using the WHO Mental Health Gap Action Programme protocols in a primary care clinic in rural Nepal. We used the Capability Opportunity Motivation-Behaviour (COM-B) implementation research framework to adapt and study the intervention. To assess implementation factors, we qualitatively studied the impact on providers' behaviour to screen, diagnose and treat mental illness. To assess clinical impact, we followed a cohort of 201 patients with moderate to severe depression and determined the proportion of patients who had a substantial clinical response (defined as $\geq 50 \%$ decrease from baseline scores of Patient Health Questionnaire (PHQ) to measure depression) by the end of the study period.

Results Providers experienced improved capability (enhanced self-efficacy and knowledge), greater opportunity (via access to counsellors, psychiatrist, medications and diagnostic tests) and increased motivation (developing positive attitudes towards people with mental illness and seeing patients improve) to provide mental healthcare. We observed substantial clinical response in 99 (49\%; $95 \% \mathrm{Cl}: 42 \%$ to $56 \%$ ) of the 201 cohort patients, with a median seven point (Q1:-9, Q3:-2) decrease in $\mathrm{PHQ}-9$ scores $(\mathrm{p}<0.0001)$.

Conclusion Using the COM-B framework, we successfully adapted and implemented CoCM in rural Nepal, and found that it enhanced providers' positive perceptions of and engagement in delivering mental healthcare. We observed clinical improvement of depression comparable to controlled trials in high-resource settings. We recommend using implementation research to adapt and evaluate CoCM in other resource-constrained settings to help expand access to high-quality mental healthcare.

\section{STRENGTHS AND LIMITATIONS OF THIS STUDY}

$\Rightarrow$ We used the Capability Opportunity MotivationBehaviour implementation research framework to adapt and study the collaborative care model (CoCM) for mental health in a real-world setting in rural Nepal.

$\Rightarrow$ We employed a mixed-methods design to study the implementation of the adapted intervention, qualitatively assess its impact on primary care provider behaviours to screen, diagnose and treat mental illness, and measured clinical outcomes among patients with depression.

$\Rightarrow$ Because the effectiveness of CoCM was already well established through at least 79 randomised controlled trials, this study focused on studying implementation factors rather than establishing effectiveness at a single site and did not have a comparison arm.

\section{INTRODUCTION}

Globally, over 322 million people suffer from depression, and depressive disorders are the largest contributor to years lived with disability. ${ }^{1}$ Despite the disproportionately high burden of depression, patients in low-income and middle-income countries (LMICs) lack access to adequate mental healthcare. In LMICs, access is impeded by both the inequitable concentration of specialised psychiatric care in urban centres with greater resources, and limited capacity among non-specialists to provide mental healthcare. ${ }^{2}$ Since the need for mental health services outweighs the capacity and number of existing specialists, shifting psychiatrists to rural areas does not address the population burden of mental illness. Interventions must expand the healthcare system's capacity to serve more patients in both urban and 
rural settings. The WHO has attempted to address this challenge via the Mental Health Gap Action Programme (mhGAP), which includes evidence-based mental health intervention guidelines for primary care providers (PCPs) ${ }^{3}$ Despite the availability of such guidelines, PCPs are often ill-equipped to deliver mental health services, exacerbating a $76 \%-85 \%$ treatment gap for patients with severe mental illness in LMICs. ${ }^{4}$

The collaborative care model (CoCM) for mental healthcare is a rigorously tested approach that addresses both the lack of specialists and high population burden of illness. Utilising PCPs and specialists in team-based care through task-sharing, CoCM has been shown to improve both mental and physical health outcomes in a review of 79 randomised controlled trials in high-resource settings. ${ }^{5-7}$ Given its demonstrated clinical effectiveness in trials through enhancing non-specialists' capacity to provide mental healthcare, CoCM can help address the large burden of mental illnesses in resource-constrained settings. However, there are limited data from implementation research studies on CoCM outside the USA. ${ }^{8}$ Despite a clear need for evidence to inform the scale-up of effective interventions, to our knowledge, no prior study has used an established implementation research framework to adapt and study CoCM in LMICs.

In Nepal, there is a pressing need to address the mental healthcare access gap in rural, resourceconstrained areas. Although the Government of Nepal committed to integrating mental health services into the primary care system through a national mental health policy in 1997, its implementation has faced numerous hurdles. ${ }^{9}$ The country's fewer than 100 psychiatrists remain concentrated in urban areas, while fewer than 10 of 75 district hospitals offer mental health services that are largely limited to dispensing psychotropic medications. ${ }^{9}$ In our prior study, PCPs in rural Nepal reported limited training, experience and self-efficacy in treating mental illness. ${ }^{2}$ They expressed a lack of capability (eg, limited psychiatric training in medical schools), opportunity (eg, no access to consult specialists or counsellors for treatment planning) or motivation (eg, many believed treating mentally ill patients was not part of their job) to provide care. ${ }^{2}$ If PCPs lack the capability, opportunity or motivation to deliver mental healthcare, the mental health treatment gap will persist. To address these challenges, we used the Capability Opportunity Motivation-Behaviour (COM-B) implementation research framework to adapt, implement and study CoCM to equip PCPs to deliver mental healthcare using mhGAP protocols at a primary care site in rural Nepal. ${ }^{10}$ Here, we present findings from a mixed-methods implementation research study to assess the implementation of the adapted CoCM, its impact on PCPs' behaviour to provide care and clinical outcomes for patients with moderate to severe depression.

\section{METHODS}

\section{Study site}

We conducted this study at the primary care clinic in a government-owned, district-level hospital in Achham, one of Nepal's poorest regions. ${ }^{11}$ Because of the weak local economy, an estimated $50 \%$ of families have at least one member working abroad. ${ }^{12}$ Since 2008 , Nyaya Health Nepal (NHN), a non-profit healthcare organisation has managed the hospital through a public-private partnership with the Ministry of Health and Population of Nepal, with support from a US-based non-profit Possible. The hospital sees over 100000 outpatient visits annually and is staffed by 15-20 PCPs, including physicians and health assistants (healthcare workers with 3 years of medical training). ${ }^{13}$ Healthcare staff use NepalEHR, an integrated electronic health record (EHR) deployed by NHN and Possible since 2015, to longitudinally document and track patient care and outcomes. ${ }^{14}$ A trained cadre of community healthcare workers augments facility-based care through home-based follow-up in the surrounding catchment areas. ${ }^{15}$ Access to mental healthcare is limited in this region, and the nearest psychiatrist is 14 hours away by road. This study was conducted in collaboration between NHN and Possible.

\section{CoCM intervention}

CoCM is a well-established, team-based, task-sharing intervention designed to provide mental healthcare at a population level. ${ }^{616}$ In this model, PCPs and behavioural health providers (eg, social workers or counsellors) at a primary care clinic evaluate patients and develop a shared treatment plan. CoCM employs proactive consultation, where a consultant psychiatrist reviews treatment plans for a panel of patients. The psychiatrist rarely provides direct patient care but supports the primary care team to ensure that patients receive high-quality, evidence-based care. Counsellors track patients' treatment plans in a registry and regularly discuss challenges and treatmentresistant cases with the psychiatrist. ${ }^{6}$

\section{Adaptations to CoCM for this study}

Most of the prior evidence for CoCM came from highincome countries. Meanwhile, several challenges need to be addressed in order to successfully implement CoCM in LMICs. ${ }^{17}$ We sought to adapt and assess CoCM in a 'real-world' setting in rural Nepal. Based on our formative study at the research site, we used COM-B, an implementation research framework based on the premise that capability, opportunity and motivation are the key factors that affect behaviour. ${ }^{10}$ We designed a set of implementation adaptations to enhance these COM-B components to encourage the PCPs' target behaviours to conduct the clinical tasks needed to deliver high-quality mental health services under CoCM: screening, diagnosis and treatment. ${ }^{10}{ }^{18}$ We provide a detailed description of the clinical workflow in online supplemental file 1 and an illustration of the pathway that patients take to access care in online supplemental file 2 . 
We also adapted the human resources to ensure local feasibility. In CoCM studies in the USA, a social worker or other specialised behavioural health professional fulfils the counsellor/care coordinator role. ${ }^{7}$ Such behavioural health workers are rare or non-existent in most LMICs. Therefore, we employed psychosocial counsellors with 3-6 months of training to conduct psychosocial evaluations and provide support using relaxation techniques, psychoeducation and basic psychotherapy. ${ }^{19}$ Furthermore, recruiting an onsite psychiatrist was infeasible and not scalable because of resource constraints. We hired an off-site psychiatrist who conducted remote panel reviews and visited the site quarterly to train the local team and directly evaluate complex cases.

Below, we describe the adaptations planned during study inception using the COM-B framework, based on a literature review and our formative studies. ${ }^{2} 132021$

1. To increase PCPs' capability (ie, having the physical and psychological capacity to engage in the target behaviours), we organised in-person training sessions led by the consultant psychiatrist. Based on WHO's mhGAP protocols, these sought to develop PCPs' interpersonal skills to build rapport with patients, use screening tools, conduct psychiatric interviews and diagnose and manage mental illness. ${ }^{3}$ We have separately reported the process and outcomes of this training. ${ }^{22}$

2. We sought to optimise the opportunity (ie, improve the physical and social contexts that make the target behaviours possible) for PCPs to provide high-quality care. As in most LMICs, clinics have little privacy and most clinic visits are only about 5 min long. ${ }^{23}$ This can severely restrict PCPs' ability to maintain patient confidentiality and conduct a full evaluation, including using mental health assessment tools (eg, PHQ9 ). Therefore, we planned to train the counsellors to support the PCPs through ensuring separate, private rooms for counselling, and tracking workloads to ensure that counsellors would spend at least $30-45 \mathrm{~min}$ per patient. We sought to enhance the social opportunity for PCPs to provide care by retaining a consultant psychiatrist for weekly supervision and for emergency consultations during the week. Additional interventions to enhance opportunity included ensuring the availability of necessary psychotropic medications in the clinic's formulary and building a reliable supply chain to the clinic's in-house pharmacy.

3. To increase motivation among PCPs (ie, receiving immediate and long-term emotional satisfaction from engaging in the target behaviours), we provided training for situations that they had previously reported as being challenging. For example, in a formative study, many PCPs had reported frustration about not knowing how to support patients who reported multiple somatic complaints without a clear physical cause. ${ }^{2}$ We hypothesised that providing training on managing such cases would enhance PCPs' motivation to provide care. Training sessions also incorporated conditions that might be considered high priority by PCPs (eg, HIV) to demonstrate the importance of mental healthcare as part of overall service delivery (eg, treating depression to improve adherence to HIV medications). The hospital's medical director would engage in persuasion by reporting mental health screening rates in monthly announcements, and publicly stating their own involvement and satisfaction in providing mental healthcare. We also planned to share each PCP's screening and treatment rates confidentially with them to provide additional persuasion to enhance motivation.

We gathered new data while implementing the study and further modified the intervention based on our preliminary findings. Figure 1 in the Results section illustrates the final version of the intervention using COM-B.

\section{Study design}

We employed a mixed-methods design to study the implementation of the adapted intervention, assess its impact on PCP behaviours to screen, diagnose and treat mental illness, and measure clinical outcomes among patients with depression. ${ }^{24}$ We employed key informant interviews (KIIs) to qualitatively assess CoCM implementation and understand its impact on PCPs' capability, opportunity and motivation to deliver mental healthcare. We shared preliminary results with PCPs to obtain suggestions and make further implementation modifications to the intervention.

To study clinical outcomes, we measured PHQ-9 scores for a cohort of patients who engaged in CoCM for at least 12 weeks. ${ }^{5}$ We used the PHQ-9 since it has been crossculturally adapted and validated in Nepal and other low-resource settings as a provider-administered scale. ${ }^{25}$ We assessed the clinical impact of the intervention by measuring the proportion of patients showing clinical response (using the commonly used definition of $\geq 50 \%$ decrease from baseline PHQ-9 score).

\section{Study participants}

We interviewed the consultant psychiatrist and purposively sampled 1-3 participants from each cadre of care providers (PCPs and counsellors) as key informants to provide in-depth feedback on CoCM implementation and its impact on provider behaviours. To assess clinical outcomes, we extracted deidentified EHR patient data over the 2-year study period (1 September 2016 to 31 August 2018). Inclusion criteria were: (i) $\geq 15$ years age (ii) receiving care through CoCM at the study site primary care clinic and living in the hospital's immediate catchment area, (iii) assessed at least once with PHQ-9 during the study period and (iv) having moderate or severe unipolar depression at baseline (ie, PHQ-9 score $\geq 10$ ). Local stakeholders noted that universal screening was infeasible and to facilitate real-world implementation, PCPs used clinical judgement based on the case descriptions in mhGAP protocols to decide which patients to screen for depression. ${ }^{3}$ We excluded patients diagnosed with bipolar affective disorder since its treatment is different from that of unipolar depression. We included comorbid conditions (such as suicidality, substance use 


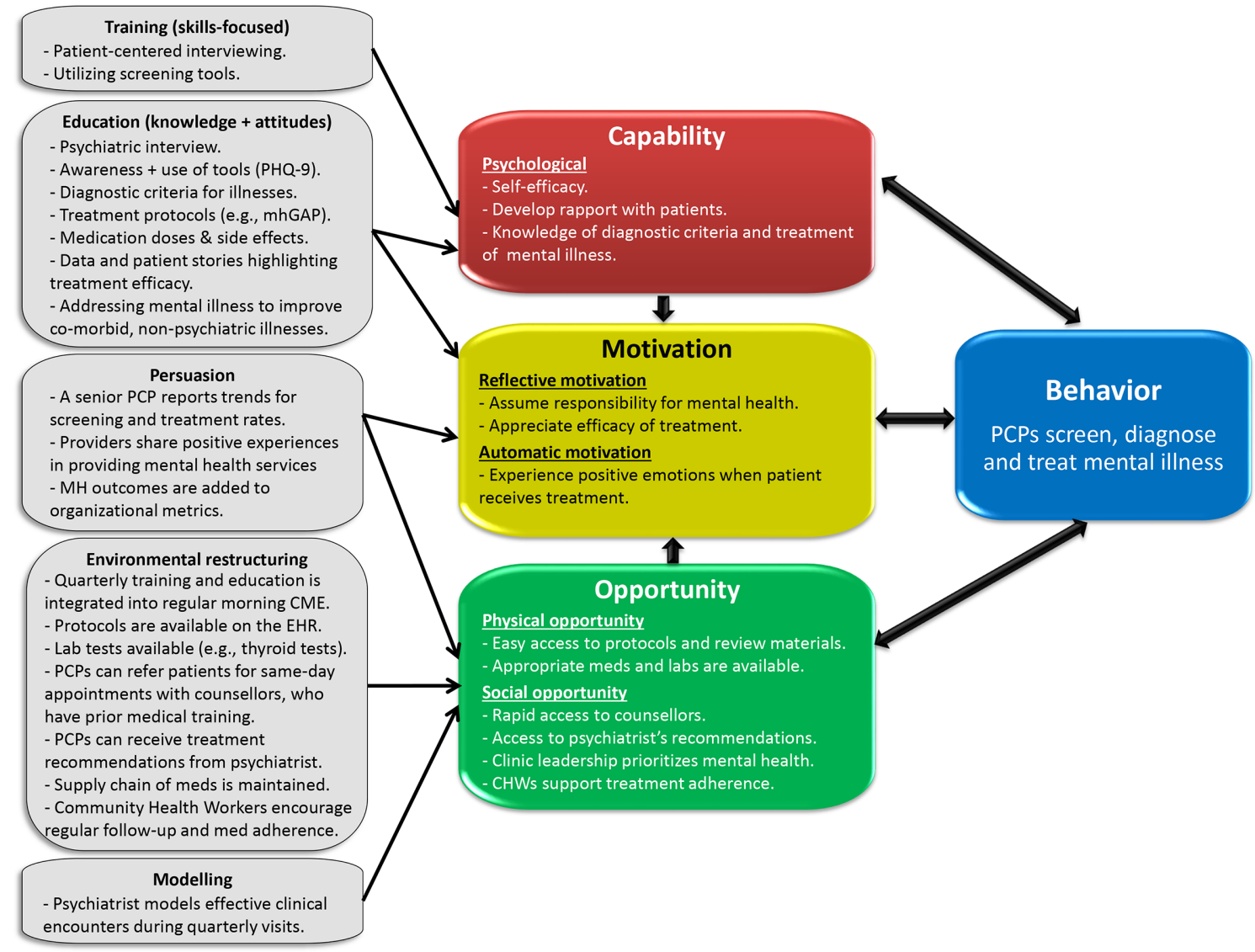

Figure 1 Final version of the CoCM intervention designed to improve PCPs' target behaviour of screening, diagnosing and treating mental illness using the COM-B implementation research framework. ${ }^{10} \mathrm{CoCM}$, collaborative care model; COM-B, Capability Opportunity Motivation-Behaviour; EHR, electronic health record; mhGAP, Mental Health Gap Action Programme; PCPs, primary care providers; PHQ, Patient Health Questionnaire.

disorder, pregnancy or psychotic features and any general medical problems), and had no other exclusion criteria. Patients needing hospitalisation for mental health conditions were offered this service at the study site's inpatient facilities. We required at least 12 weeks between patients' baseline and most recent PHQ-9 scores for inclusion in the analysis cohort. This provided sufficient time to initiate medications, titrate them to a therapeutic dose and allow the requisite 4-6 weeks for antidepressant effect. Figure 2 summarises the enrolment of patients into the cohort.

\section{Data collection}

PR conducted nine KIIs with the providers in Nepali to gain insights on: overall experience with the intervention, challenges in implementation, strategies to overcome challenges, impact on their knowledge and attitudes and any additional feedback. Eight of these interviews were conducted privately in the clinic and one via a private videoconference. Additionally, during the intervention period, PR conducted seven KIIs with the providers for ongoing feedback to assess and adapt the intervention's implementation.

We extracted deidentified patient data collected during routine care provision from the EHR to assess clinical outcomes. When entering patient data, counsellors clicked on responses to the nine PHQ questions, each corresponding to a numeric score and entered a 'total PHQ-9 score' based on patient responses. We computed total PHQ-9 scores by adding the numeric scores associated with the nine questions. For patient records with missing responses to one or more PHQ questions, we used aggregate, clinician-entered 'total PHQ-9' scores instead. We also used EHR data to report on patient demographics, the number of patients assessed using the PHQ-9 tool and the proportion of patients with moderate to severe unipolar depression who were prescribed antidepressants.

\section{Data analysis}

PR transcribed and translated audio recordings of KIIs from Nepali to English and analysed them using thematic analysis guided by the COM-B framework, while also coding other emergent themes. ${ }^{26} \mathrm{PR}$ and BA discussed themes until arriving at a consensus. To analyse clinical outcomes, we compared each patient's most recent PHQ-9 score within the observation period (after at least 12 weeks from baseline) to their baseline PHQ-9 score. We computed $95 \%$ CIs for the proportion of patients demonstrating clinical response (ie, $\geq 50 \%$ reduction from baseline). Since data were non-normally distributed, we used the Wilcoxon signed-rank test to assess the median PHQ-9 score change for patients. We used SAS software, V.9.4 for quantitative analyses. ${ }^{27}$ 
Enrollment

Patients screened using PHQ-9 during
study period ( $\mathbf{n = 8 6 2}$ )
(i) Age 15y or older (ii) from hospital's
catchment area

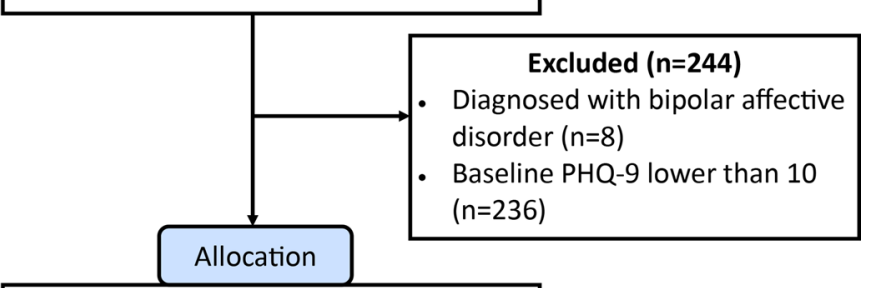

\begin{tabular}{|l|}
\hline \multicolumn{1}{|c|}{ Received CoCM intervention $(\mathbf{n}=\mathbf{6 1 8})$} \\
Moderate to severe unipolar depression at \\
baseline \\
- Moderate depression, PHQ-9 score 10 \\
to $15(n=305)$ \\
- Severe depression, PHQ-9 score $>10$ \\
$(n=313)$
\end{tabular}

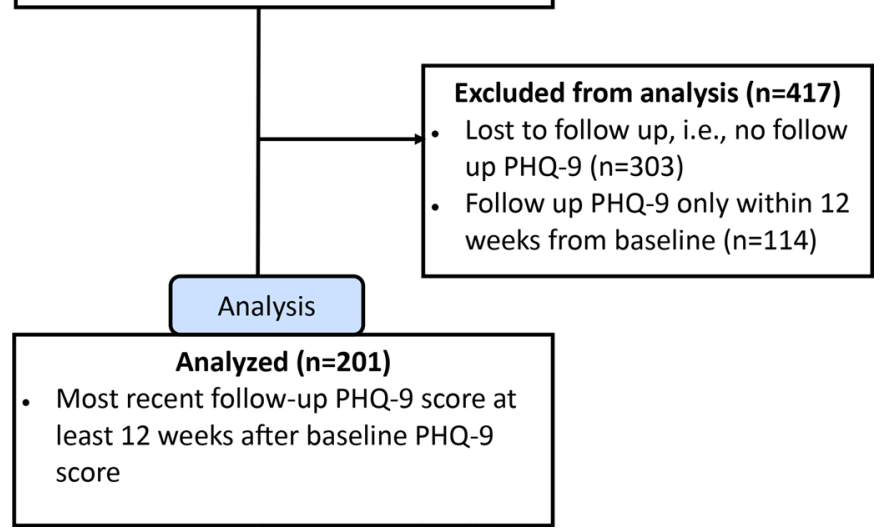

Figure 2 Patient enrolment in study cohort.

\section{RESULTS}

\section{Intervention implementation and impact on provider behaviour}

We implemented CoCM at a large primary care clinic in a district-level hospital between February 2016 and May 2018. Typically, 15 PCPs simultaneously see patients in the clinic but due to high turnover in the region, at least 93 PCPs received the mental health training during the study period. The counsellors and psychiatrist did not turnover during the study period. Panel reviews, where the offsite psychiatrist reviewed cases with the primary care team, were usually conducted weekly. Below, we summarise the providers' (five PCPs, one psychiatrist and three counsellors) perspectives on the intervention's impact on their capability, opportunity and motivation to provide quality mental healthcare, supported with exemplary quotes.

\section{Capability}

All PCPs reported improved clinical knowledge, selfefficacy and increased ability to identify and treat mental illness. One noted:

We had limited knowledge (about mental illness) and were scared that those patients would harm us.
Now we can take care of them, we know how to talk to them and the fear has decreased. (PCP 5)

According to the consultant psychiatrist, coaching the PCPs was more important than didactic lectures during on-site training sessions in enhancing their capability:

We have two types of training-(a) classroom based... and (b) coaching. Coaching is a unique approach in our setting... my role is to encourage PCPs to evaluate patients (instead of directly evaluating patients), observe, guide, and provide feedback. (Psychiatrist)

\section{Opportunity}

Most participants observed that the team-based approach across clinicians increased opportunities to provide care while improving their competency. One stated:

Before, we did not have counselors. There used to be a single trained focal person who dealt with all patients with mental illness. We also did not have many patients at the (clinic). We did not know how to provide high-quality care either, now all that has improved. (PCP 5)

When asked about their working relationship with the psychiatrist, most PCPs found the psychiatrist accessible, and the panel reviews helpful in instilling confidence about the quality of care. According to one PCP:

The relationship is remote but good. We address challenges and confusion in panel reviews. In case of emergencies, we contact him via counselors on his phone. He is readily available to support. (PCP 1)

According to the psychiatrist:

Geographically, we are far but we try to turn on the videos whenever possible and that keeps us connected. (Psychiatrist)

Others preferred working in closer proximity with the psychiatrist and noted challenges in remote consultation, while acknowledging the resource constraints. The counsellors reported trying to maximise face-to-face interaction with the psychiatrist during on-site visits. One of them shared:

I wish we were working together (in person). There are challenges with remote consultation, sometimes, (phone and internet) connection is bad, the patient flow at the (clinic) is high, and we have to reschedule the panel reviews. (Counselor 2)

The consultant psychiatrist emphasised the team-based approach where research, clinical staff and counsellors contributed to shaping the programme and supporting its success:

The support from other teams has been substantial in streamlining mental healthcare. From planning my on-site visits, developing the training, presenting cases in panel reviews, increasing participation during 
panel reviews, constant monitoring, and feedback... (Psychiatrist)

\section{Motivation}

All PCPs attributed a positive change in their attitude about providing care for patients with mental illness. A counsellor mentioned feeling motivated when patients responded to treatment:

Patients used to share how their conditions would not improve despite taking the many medicines bought from (private, usually unlicensed medical personnel). Affirmations from patients who never imagined getting better with counseling motivate me. What we are doing for patients with mental illness is optimal. (Counsellor 2)

\section{Final intervention}

We now describe the additional adaptations we made to the original intervention using COM-B, based on qualitative data collected throughout its implementation.

\section{Capability}

1. Quarterly onsite visits: Onsite training sessions increased providers' capability to identify and treat mental illness. In trying to determine an appropriate frequency for the consultant psychiatrist's visits, we found that quarterly, week-long visits were most feasible and effective. Per PCPs' feedback, anything more frequent would be infeasible and less frequent would impede continuity. Based on this, the psychiatrist conducted onsite quarterly trainings eight times $(100 \%$ of expected) during the study period.

2. Providing psychiatrist recommendations along with a rationale: After the counsellors conducted a panel review, they entered the psychiatrist's recommendations in the EHR. During CoCM implementation, PCPs suggested that if the psychiatrist included the rationale behind each suggestion, they would be more likely to accept and incorporate it. For example, instead of simply saying, 'increase fluoxetine to $40 \mathrm{mg}$ daily', a rationale such as 'patient has been on $20 \mathrm{mg}$ for 2 months and her PHQ-9 scores have not improved so we should try a higher dose' was preferable. Based on this feedback, we edited the EHR template and the counsellors began including the psychiatrist's rationale alongside the recommendations. In subsequent KIIs, PCPs confirmed that this helped them identify specific content areas they needed to work on (eg, appropriate antidepressant titration schedule) and enhanced their motivation to follow the psychiatrist's recommendations.

\section{Opportunity}

1. Employing counsellors with prior medical training: The counsellors we initially recruited had 3-6 months of psychosocial training, and two out of three had also received medical training. We found that in weekly panel reviews, the counsellor without medical training struggled to accurately document and convey medical information such as recommended laboratory tests (eg, check Thyroid Stimulating Hormone level) or medication changes (eg, increase fluoxetine to $20 \mathrm{mg}$ and provide 5-day short course of clonazepam during titration). Thereafter, we changed our recruitment strategy to employ counsellors with some medical background (eg, completion of a 3-year medical programme, similar to a physician's assistant programme in the USA) in addition to psychosocial training, to enhance collaboration with PCPs.

2. Designating a local mental health programme supervisor: We noted that the research coordinator was becoming the primary go-to person for all issues related to mental healthcare delivery, threatening sustainability after the study period. The clinical team nominated a senior onsite clinician to be the primary programme supervisor. This role entailed directly supervising the counsellors, coordinating the psychiatrist's visits, highlighting the programme's success via internal team communication platforms, planning the training curriculum and troubleshooting any challenges.

3. Process for emergency consultations with the psychiatrist: PCPs' access to the psychiatrist for urgent questions that could not wait until the weekly panel review was an important consideration for enhancing their opportunity. We introduced a process for such emergency consultations. PCPs and counsellors first discussed the case with the onsite mental health supervisor. If this team was unable to manage the case, the counsellors would contact the psychiatrist via a designated telephone. This provided PCPs the opportunity they requested without overwhelming the offsite psychiatrist with numerous calls from unknown phone numbers throughout the week.

\section{Motivation}

1. Orienting staff members to team-based care: Prior to the intervention, counsellors were not part of the primary care system while PCPs independently provided care without any coordination. During the early implementation phase, PCPs were concerned about collaborating with counsellors, who they perceived as subordinates since counsellors do not prescribe medications. Similarly, counsellors felt their contributions were unrecognised because they were not prescribers. They also reported discomfort when sharing suggestions or disagreeing with the PCPs. Despite training clinicians about the team-based approach in CoCM, communication challenges and role-confusion arose, especially among new PCPs. These were exacerbated by the high clinician turnover and use of the EHR as the primary communication and care coordination tool. Initially, these factors negatively affected PCPs' opportunity and motivation to provide care. To help establish counsellors as an integral part of the intervention and facilitate face-to-face communication with PCPs, we initiated a dedicated mental health 
onboarding programme. This positioned existing counsellors to orient all new PCPs to CoCM, its workflow and everyone's roles. During subsequent KIIs, counsellors reported feeling established as key members of the clinical team, and noted decreased hierarchy and role confusion.

2. Introducing mental health integrated case conference: Initially, various providers (PCPs, counsellors and others) often did not discuss cases and blamed the other group for errors. To address this, we implemented a monthly conference where a representative from each team selected challenging cases that would benefit from group discussion. Presenters received guidance to employ systems-thinking to discuss patient history, treatment summary and barriers to care. This served as a platform for interdisciplinary communication to generate ideas for improved care delivery. Facilitating a better understanding of other cadres increased providers' motivation to provide collaborative care.

\section{Clinical outcomes}

Over the 2-year study period, 862 unique patients $(\geq 15$ years) from the hospital's immediate catchment area were assessed for depression at the primary care clinic using the PHQ-9, and accounted for 2309 total clinic visits. These 862 patients had 2.7 mean $( \pm \mathrm{SD}$ : 2.9) clinic visits with documented PHQ-9 scores. Among patients with a follow-up PHQ-9 score $(\mathrm{n}=403,47 \%)$, the median duration between the baseline and most recent PHQ-9 scores was 168 days (Q1: 60, Q3: 392).

At baseline, most patients had severe depression $(\mathrm{n}=313,37 \%)$ or moderate depression $(\mathrm{n}=305,36 \%)$, excluding those with bipolar affective disorder $(n=8)$. Among patients with moderate to severe unipolar depression at baseline $(\mathrm{n}=618), 526(85 \%)$ were prescribed an antidepressant during the study. Table 1 summarises the demographic characteristics of the 618 patients. We excluded from the analysis cohort 303 (49\%) patients with moderate to severe unipolar depression who had no follow-up PHQ-9 score and 114 (18\%) patients with a follow-up PHQ-9 administered before the requisite 12-week lead time.

Table 1 Characteristics of patients with moderate to severe unipolar depression at baseline

\begin{tabular}{|c|c|}
\hline Characteristic & Patients $(n=618)$ \\
\hline Age, mean $\pm S D$ & $35.1 \pm 12$ \\
\hline \multicolumn{2}{|l|}{ Sex, n (\%) } \\
\hline Female & $502(81 \%)$ \\
\hline Male & $116(19 \%)$ \\
\hline \multicolumn{2}{|l|}{ Caste, n (\%) } \\
\hline $\begin{array}{l}\text { Brahmin/Chhetri ('high' } \\
\text { caste) }\end{array}$ & 332 (54\%) \\
\hline Non-Brahmin/Chhetri & $286(46 \%)$ \\
\hline
\end{tabular}

Table 2 Comparing characteristics of cohort patients to other patients with moderate/severe unipolar depression excluded from analysis

\begin{tabular}{|c|c|c|c|}
\hline Characteristic & $\begin{array}{l}\text { Excluded from } \\
\text { analysis }(n=417)\end{array}$ & $\begin{array}{l}\text { Included in } \\
\text { cohort }(n=201)\end{array}$ & $P$ value \\
\hline Age, mean $\pm S D$ & $34.4 \pm 12$ & $36.6 \pm 11$ & 0.02 \\
\hline $\begin{array}{l}\text { Baseline PHQ-9 } \\
\text { score, mean } \pm \text { SD }\end{array}$ & $14.5 \pm 2$ & $14.7 \pm 3$ & 0.44 \\
\hline \multicolumn{4}{|l|}{ Sex, n (\%) } \\
\hline Female & $330(79 \%)$ & 172 (86\%) & \multirow{2}{*}{0.05} \\
\hline Male & $87(21 \%)$ & $29(14 \%)$ & \\
\hline \multicolumn{4}{|l|}{ Caste, n (\%) } \\
\hline $\begin{array}{l}\text { Brahmin/Chhetri } \\
\text { ('high' caste) }\end{array}$ & $236(57 \%)$ & $96(48 \%)$ & \multirow{2}{*}{0.04} \\
\hline $\begin{array}{l}\text { Non-Brahmin/ } \\
\text { Chhetri }\end{array}$ & $181(43 \%)$ & $105(52 \%)$ & \\
\hline
\end{tabular}

Table 2 compares the baseline characteristics of patients included in the analysis cohort with those excluded using appropriate bivariate tests. The mean baseline PHQ-9 score was not significantly different between the two groups $(\mathrm{p}=0.44)$. Patients included in analysis, however, were more likely to be about 2 years older, female, and of non-Brahmin/Chhetri (marginalised) castes compared with those not included. Of all patient encounters with PHQ-9 scores $(n=2309), 20(<1 \%)$ were missing responses to some of the nine PHQ questions. For these encounters, we used the 'total PHQ-9 score' entered by clinicians.

Of the 201 patients in the analysis cohort, $99(49 \%$, 95\% CI: $42 \%$ to $56 \%$ ) demonstrated substantial clinical response, that is, their most recent PHQ-9 score was at least $50 \%$ lower than their baseline score. A subset of these patients $(n=25,12 \%$ of the cohort) showed remission in depression, that is, their PHQ-9 score dropped to below 5 at their most recent follow-up (95\% CI: $8 \%$ to $17 \%$ ). The median change in PHQ-9 score in this cohort was -7 points (Q1: -9, Q3: -2) which was both statistically significant $(\mathrm{p}<0.0001)$ at $\alpha=0.05$ and clinically meaningful.

\section{DISCUSSION}

Despite the wide dissemination of mhGAP protocols in numerous countries, the gap between knowledge and practice is substantial in global mental health. ${ }^{38} \mathrm{CoCM}$ holds substantial promise in addressing this gap, as demonstrated by evidence from at least 79 randomised controlled trials. ${ }^{5}$ Our findings highlight that, when adapted and implemented using a structured implementation research framework, CoCM changed care providers' perception and delivery of mental healthcare at the intervention site in rural Nepal. We observed substantial clinical response (49\%, 95\% CI: $42 \%$ to $56 \%$ ) in our patient cohort, which is comparable to that found in meta-analyses of 12-24 month CoCM trials from highincome countries. ${ }^{5}$ 
As described by the COM-B framework, increasing the PCPs' capability via training, providing opportunities (eg, establishing access to a consultant psychiatrist) and enhancing motivation (eg, witnessing improved patient outcomes) helped achieve the target behaviour of screening, diagnosing and treating mental illness. As mental health services became available in our primary care system, a programmatically meaningful number of patients (862 over 2 years) were assessed using PHQ-9 in a setting where patients previously had limited or no access to quality mental healthcare. This likely included patients who previously sought tertiary care or did not seek care because of barriers like distance and stigma. PCPs at the facility screened patients with suspected mental illness, if their presentation was similar to the cases described in mhGAP training. These factors may explain the large proportion $(73 \%)$ of patients with high or moderate symptoms of depression in our study. Our prior study at the same site indicated that almost all patients with depression and anxiety disorders were previously receiving only vitamins, painkillers or no treatment. ${ }^{2}$ In contrast, during this study, patients who were prescribed pharmacotherapy ( $85 \%$ of all cases with moderate/severe depression) received evidence-based treatment such as antidepressants and counselling.

Initial qualitative data from KIIs revealed challenges in team-based work, especially since integrating counsellors and PCPs in providing mental healthcare is uncommon. These initial challenges, including role confusion, are common barriers in implementing CoCM. ${ }^{28}$ Introducing interventions such as mental health onboarding and an interdisciplinary conference helped overcome these challenges. PCPs gradually valued the counsellors' role and saw them as experts in their field. This suggests the value of training all care providers in mental health instead of colocating a single, designated mental healthcare provider in resource-limited settings. ${ }^{29}$

Our study has several limitations. Because of resource limitations, we could only focus on a single site. Since CoCM is a facility-wide intervention, our study did not have a control arm to avoid contamination. Although this limits inferential results, our primary research question for CoCM focused on its implementation and overall clinical impact, since CoCM already has substantial evidence for clinical effectiveness from controlled studies. ${ }^{5} 30 \mathrm{~A}$ single site study allowed us to generate evidence within the available resources, in a real-world, government facility operated under a public-private partnership. A future pragmatic trial can test the final version of the CoCM intervention in multiple primary care clinics. Future work should also elucidate payment mechanisms for CoCM feasible within the particular local and national policy and fiscal context.

Another limitation was that $49 \%$ of patients with a baseline PHQ-9 score had no follow-up score. While retention in real-world studies of depression is always a challenge, this might be partially explained by the high rate of outmigration from the catchment area. However, cohort patients had similar baseline scores to those with no or less than 12 weeks of follow-up despite other differences. Furthermore, the observed loss to follow-up aligned with the $50 \%$ rate of attrition we had anticipated from empirical data and high migration rates at the outset. Nevertheless, this finding demonstrates the importance of close follow-up with patients to ensure treatment engagement for depression. We are currently conducting a study to address this challenge in rural Nepal through engaging community health workers. ${ }^{31}$ While a future study with an appropriate comparison group could better assess the impact of the intervention on clinical outcomes, a programmatically meaningful proportion $(49 \%)$ of cohort patients demonstrated clinical response. Since we did not collect extensive data on potential confounders, factors besides the intervention, such as socioeconomic status and other treatments may have also affected patient outcomes. As is common in mental health studies, regression to the mean, whereby patients may experience spontaneous improvement in their symptoms without any intervention, may have contributed to the observed results. However, regression to the mean is more likely to be observed in those with milder depressive symptoms. ${ }^{32}$ This was at least partially mitigated since we had excluded patients who were most likely to regress to the mean through our inclusion criteria for baseline PHQ $>9$.

\section{CONCLUSION}

Despite the limitations, our findings suggest that an adapted CoCM enhanced providers' perception and delivery of mental healthcare in our setting, and we observed improved clinical outcomes in patients with moderate or severe depression. We recommend adapting and evaluating CoCM using principles of implementation research in similar rural and resource-constrained settings to help expand access to high-quality mental healthcare.

\section{Author affiliations}

${ }^{1}$ Nyaya Health Nepal, Kathmandu, Nepal

${ }^{2}$ Possible, Kathmandu, Nepal

${ }^{3}$ Arnhold Institute for Global Health, Icahn School of Medicine at Mount Sinai, New York, New York, USA

${ }^{4}$ Possible, New York, New York, USA

${ }^{5}$ Department of Psychiatry, BP Koirala Institute of Health Sciences, Dharan,

Kathmandu, Nepal

${ }^{6}$ Department of Global Health, University of Washington, Seattle, Washington, USA ${ }^{7}$ Internal Medicine, Hurley Medical Center, Flint, Michigan, USA

${ }^{8}$ Global Health, University of Washington, Seattle, Washington, USA

${ }^{9}$ Health Services, Los Angeles County Department of Health Services, Los Angeles,

California, USA

${ }^{10}$ University of California Los Angeles David Geffen School of Medicine, Los Angeles, California, USA

${ }^{11}$ Division of Global Health Equity, Brigham and Women's Hospital Department of Medicine, Boston, Massachusetts, USA

${ }^{12}$ Department of Psychology, Wheaton College, Wheaton, Massachusetts, USA

${ }^{13}$ University of New South Wales School of Public Health and Community Medicine, Sydney, New South Wales, Australia

${ }^{14}$ Psychiatry and Behavioral Sciences, University of California San Francisco, San Francisco, California, USA

Correction notice This article has been corrected since it first published. 'Srijana Shrestha' affiliation has been updated. 
Twitter Pragya Rimal @rimal_pragya and Duncan Maru @duncanmaru

Acknowledgements We thank Margaret Handley and Adithya Cattamanchi for their support in helping apply the COM-B framework to mental health services, as part of the UCSF Clinical \& Translational Science Institute (CTSI) funded by NIH (UL1 TR001872). We also thank Lisa Sullivan from Boston University School of Public Health for her advice on statistical methods, and Jerome Galea and Courtney Yuen from Harvard Medical School for their mentorship in scientific writing. We wish to express our appreciation to the Nepal Ministry of Health and Population for their continued efforts to improve the public-sector healthcare system in rural Nepal. Finally, we are deeply indebted to the community and hospital staff, whose commitment to serving patients and dedication to improving the quality of healthcare in rural Nepal continues to inspire us.

Contributors PR and NC contributed equally to this paper as joint first authors. $\mathrm{PR}, \mathrm{MB}, \mathrm{DC}, \mathrm{DM}$ and $\mathrm{BA}$ led the conception and design of the study. PR, PA, MB, $B B, S K D, B G, P G, T K G, B K, J R, S S w$ and BA helped develop and implement the intervention. PR, PA, MB, SKD, BG, SH, BK, SSw and BA managed the project. PR, NC, RM, SP and AR extracted and managed study data, with PR leading the qualitative data analysis and NC conducting statistical analyses with input from BA, $\mathrm{DM}, \mathrm{SH}$ and DC. PR and NC led the initial draft of the manuscript with input from all authors. VN, SSa, DS, RS, SSh, AT, PT and RW contributed technical expertise to the manuscript. All authors further drafted, reviewed and approved the final manuscript.

Funding National Institute of Mental Health [grant number R25MH060482] and Harvard Medical School Center for Global Health Delivery, Dubai [027562-746845-0201].

Competing interests PR, PA, MB, BB, BG, PG, TKG, BK, RM, SP, JR, SSw and PT were employed by, and NC, DC, SH, DM, AR, DS, RS, SSa, SSh, AT and BA work in partnership with a non-profit healthcare company (Nyaya Health Nepal, with support from the US-based non-profit, Possible) that delivers free healthcare in rural Nepal using funds from the Government of Nepal and other public, philanthropic and private foundation sources. MB is a faculty at BP Koirala Institute of Health Sciences, Dharan, Nepal. NC, SH and DM are employed by, and DC, DM and SSa are faculty members at a private medical school (Icahn School of Medicine at Mount Sinai). DC is a faculty member at, DC and SH are employed part-time by and SH is a graduate student at a public university (University of Washington). SKD is a resident at an academic medical centre (Hurley Medical Center) that receives revenue through private sector fee-for-service medical transactions and a charitable private foundation. TKG is a fellow with a bidirectional fellowship program (HEAL Initiative) that is affiliated with a public university (University of California, San Francisco) that receives funding from public, philanthropic and private foundation sources. DM and BA are members on Possible's Board of Directors, for which they receive no compensation. VN is employed at a public university (University of California, Los Angeles). DS and RS are employed at an academic medical centre (Brigham and Women's Hospital) that receives public sector research funding, as well as revenue through private sector fee-for-service medical transactions and private foundation grants. DS and RS are faculty members at a private medical school (Harvard Medical School). DS is employed at an academic research centre (Ariadne Labs) that is jointly supported by an academic medical center (Brigham and Women's Hospital) and a private university (Harvard TH Chan School of Public Health) via public sector research funding and private philanthropy. RS is employed at an academic medical centre (Massachusetts General Hospital) that receives public sector research funding, as well as revenue through private sector fee-for-service medical transactions and private foundation grants. SSh is a faculty member at a private college (Wheaton College). PT is a graduate student at a public university (University of New South Wales). BA is a faculty member at a public university (University of California, San Francisco). All authors have read and understood BMJ Open's policy on declaration of interests, and declare that we have no competing financial interests. The authors do, however, believe strongly that healthcare is a public good, not a private commodity.

Patient consent for publication Not required.

Ethics approval This study was approved by the Nepal Health Research Council (Reg. No. 204/2016), University of California, San Francisco (Reference 171608), and Brigham and Women's Hospital (2016P001858/PHS) institutional review boards. Since obtaining feedback from providers was part of the intervention, PR obtained informed verbal consent for key informant interviews.

Provenance and peer review Not commissioned; externally peer reviewed.

Data availability statement Data are available upon reasonable request. Deidentified EHR data, statistical analysis plans, and statistical code are available upon reasonable request to authors.
Supplemental material This content has been supplied by the author(s). It has not been vetted by BMJ Publishing Group Limited (BMJ) and may not have been peer-reviewed. Any opinions or recommendations discussed are solely those of the author(s) and are not endorsed by BMJ. BMJ disclaims all liability and responsibility arising from any reliance placed on the content. Where the content includes any translated material, BMJ does not warrant the accuracy and reliability of the translations (including but not limited to local regulations, clinical guidelines, terminology, drug names and drug dosages), and is not responsible for any error and/or omissions arising from translation and adaptation or otherwise.

Open access This is an open access article distributed in accordance with the Creative Commons Attribution Non Commercial (CC BY-NC 4.0) license, which permits others to distribute, remix, adapt, build upon this work non-commercially, and license their derivative works on different terms, provided the original work is properly cited, appropriate credit is given, any changes made indicated, and the use is non-commercial. See: http://creativecommons.org/licenses/by-nc/4.0/.

ORCID iDs

Pragya Rimal http://orcid.org/0000-0003-4442-4609

Duncan Maru http://orcid.org/0000-0002-5923-6910

\section{REFERENCES}

1 World Health Organization. Depression and other common mental disorders. In: Global health estimates. 24, 2017.

2 Acharya B, Hirachan S, Mandel JS, et al. The mental health education gap among primary care providers in rural Nepal. Acad Psychiatry 2016;40:667-71.

3 World Health Organization. mhGAP intervention guide for mental, neurological and substance use disorders in non-specialized health settings: version 2.0. Geneva, 2016

4 World Health Organization. Comprehensive mental health action plan 2013-2020, 2013.

5 Archer J, Bower P, Gilbody S, et al. Collaborative care for depression and anxiety problems. Cochrane Database Syst Rev 2012;10:CD006525

6 Raney LE. Integrating primary care and behavioral health: the role of the psychiatrist in the collaborative care model. Am J Psychiatry 2015;172:721-8.

7 Goodrich DE, Kilbourne AM, Nord KM, et al. Mental health collaborative care and its role in primary care settings. Curr Psychiatry Rep 2013;15:383-83.

8 Patel V, Saxena S, Lund C. The lancet commission on global mental health and sustainable development. Lancet 2018.

9 Luitel NP, Jordans MJ, Adhikari A, et al. Mental health care in Nepal: current situation and challenges for development of a district mental health care plan. Confl Health 2015;9:3.

10 Michie S, van Stralen MM, West R. The behaviour change wheel: a new method for characterising and designing behaviour change interventions. Implement Sci 2011;6:42.

11 Government of Nepal National Planning Commission, United Nations Development Programme. Nepal human development report 2014 : beyond geography, unlocking human potential. Kathmandu, Nepal, 2014.

12 Johnson DC, Lhaki P, Bhatta MP, et al. Spousal migration and human papillomavirus infection among women in rural Western Nepal. Int Health 2016;8:261-8.

13 Acharya B, Tenpa J, Thapa P, et al. Recommendations from primary care providers for integrating mental health in a primary care system in rural Nepal. BMC Health Serv Res 2016;16:492.

14 Raut A, Yarbrough C, Singh V, et al. Design and implementation of an affordable, public sector electronic medical record in rural Nepal. $J$ Innov Health Inform 2017;24:186-95.

15 Citrin D, Thapa P, Nirola I, et al. Developing and deploying a community healthcare worker-driven, digitally- enabled integrated care system for municipalities in rural Nepal. Healthc 2018;6:197-204.

16 Unützer J, Katon W, Callahan $\mathrm{CM}$, et al. Collaborative care management of late-life depression in the primary care setting: a randomized controlled trial. JAMA 2002;288:2836-45.

17 Acharya B, Ekstrand M, Rimal P, et al. Collaborative care for mental health in low- and middle-income countries: a who health systems framework assessment of three programs. Psychiatr Serv 2017;68:870-2

18 Hartmann CW, Mills WL, Pimentel CB, et al. Impact of intervention to improve nursing home resident-staff interactions and engagement. Gerontologist 2018;58:e291-301. 
19 Jordans MJ, Tol WA, Sharma B. Training psychosocial counselling in Nepal: content review of a specialised training programme. Intervention 2003;1.

20 Acharya B, Basnet M, Rimal P, et al. Translating mental health diagnostic and symptom terminology to train health workers and engage patients in cross-cultural, non-english speaking populations. Int J Ment Health Syst 2017;11:62.

21 Acharya B, Maru D, Schwarz R, et al. Partnerships in mental healthcare service delivery in low-resource settings: developing an innovative network in rural Nepal. Global Health 2017;13:2.

22 Acharya B, Tenpa J, Basnet M, et al. Developing a scalable training model in global mental health: pilot study of a video-assisted training program for generalist clinicians in rural Nepal. Glob Ment Health 2017;4:e8.

23 Irving G, Neves AL, Dambha-Miller $\mathrm{H}$, et al. International variations in primary care physician consultation time: a systematic review of 67 countries. BMJ Open 2017;7:e017902.

24 Curran GM, Bauer M, Mittman B, et al. Effectiveness-implementation hybrid designs: combining elements of clinical effectiveness and implementation research to enhance public health impact. Med Care 2012;50:217-26.

25 Kohrt BA, Luitel NP, Acharya P, et al. Detection of depression in low resource settings: validation of the patient health questionnaire
(PHQ-9) and cultural concepts of distress in Nepal. BMC Psychiatry 2016:16:1-14.

26 Ulin PR, Robinson ET, Tolley EE. Qualitative methods in public health : a field guide for applied research. San Francisco, CA: Jossey-Bass, 2005.

27 SAS. version 9.4 [program. Cary, NC: SAS Institute Inc, 2013.

28 Wood E, Ohlsen S, Ricketts T. What are the barriers and facilitators to implementing collaborative care for depression? A systematic review. J Affect Disord 2017;214:26-43.

29 Patel V, Belkin GS, Chockalingam A, et al. Grand challenges: integrating mental health services into priority health care platforms. PLoS Med 2013;10:e1001448.

30 Gilbody S, Bower P, Fletcher J, et al. Collaborative care for depression: a cumulative meta-analysis and review of longer-term outcomes. Arch Intern Med 2006;166:2314-21.

31 Acharya B, Maru D. Acceptability and feasibility of communitybased mHealth motivational interviewing tool for depression (COMMIT-D) to improve adherence to treatment. National Institutes of Health Research Portfolio Online Reporting Tools (NIH RePORT), 2019.

32 Whiteford HA, Harris MG, McKeon G, et al. Estimating remission from untreated major depression: a systematic review and metaanalysis. Psychol Med 2013;43:1569-85. 